\title{
LOCALLY COMPACT GROUPS WHICH ARE AMENABLE AS DISCRETE GROUPS
}

\author{
CHING $^{\mathrm{CHOU}^{1}}$
}

\begin{abstract}
In the courses of their independent studies of the existence of invariant means which are not topologically invariant, E. Granirer and W. Rudin have considered several properties of a locally compact group $G$ which are satisfied if $G$ is amenable as a discrete group. By applying a result of $J$. Rosenblatt (together with ideas of Granirer and Rudin) we show some of these properties on $G$ imply that $G$ is amenable as a discrete group.
\end{abstract}

Let $G$ be a locally compact group with a fixed left Haar measure $\lambda$. As usual, if $G$ is compact then $\lambda$ is assumed to be normalized, i.e., $\lambda(G)=1$. Let $L^{\infty}(G)$ be the Banach algebra of bounded real-valued Borel functions on $G$ with the $\lambda$-essential supremum norm $\|\cdot\|_{\infty}$. For $f \in L^{\infty}(G)$ and $x \in G$, $l_{x} f \in L^{\infty}(G)$ is defined by $\left(l_{x} f\right)(y)=f(x y), y \in G$. For $\varphi \in L^{\infty}(G)^{*}$ and $x \in G, L_{x} \varphi \in L^{\infty}(G)^{*}$ is defined by $\left(L_{x} \varphi\right)(f)=\varphi\left(l_{x} f\right), f \in L^{\infty}(G)$. Let $\operatorname{LIM}$ (or $\operatorname{LIM}(G)$ ) be the set of left invariant means on $L^{\infty}(G)$, i.e., $\operatorname{LIM}=$ $\left\{\mu \in L^{\infty}(G)^{*}:\|\mu\|=1, \mu \geqslant 0\right.$ and $L_{x} \mu=\mu$ for $\left.x \in G\right\}$. $G$ is said to be amenable if $\operatorname{LIM} \neq \varnothing$. Every compact group is amenable since $\lambda \in$ LIM.

Let $\mathscr{D}$ be the maximal ideal space of $L^{\infty}(G)$. With the Gelfand topology, OD is a compact Hausdorff space. The Gelfand transform ${ }^{\wedge}$ is an isometry of $L^{\infty}(G)$ onto $C(\mathscr{D})$, the algebra of real-valued continuous functions on $\mathscr{D}$ with supremum norm. Note that if $\theta \in \mathscr{D}$ then $L_{x} \theta \in \mathscr{D}$ and each $\mu \in$ LIM can be identified with a $G$-invariant probability measure $\hat{\mu}$ on $\mathscr{D}: \hat{\mu}(\hat{f})=$ $\mu(f), f \in L^{\infty}(G)$. For $\theta \in \mathscr{D}$, let $\rho_{\theta}: L^{\infty}(G) \rightarrow l^{\infty}(G)$ be defined by

$$
\left(\rho_{\theta} f\right)(x)=\theta\left(l_{x} f\right), \quad x \in G .
$$

Here $l^{\infty}(G)=L^{\infty}\left(G_{d}\right)$ where $G_{d}$ is the discrete version of $G$. Note that $\left\|\rho_{\theta}\right\| \leqslant 1, \rho_{\theta} \geqslant 0$ and $\rho_{\theta}\left(l_{x} f\right)=l_{x}\left(\rho_{\theta} f\right)$, for $x \in G$ and $f \in L^{\infty}(G)$.

The purpose of this paper is to prove the following.

THEOREM. Let $G$ be a $\sigma$-compact locally compact group. Then the following conditions are equivalent:

(1) $G_{d}$ is amenable;

(2) $G$ is amenable and, for each $f \in L^{\infty}(G)$,

Received by the editors October 13, 1978.

AMS (MOS) subject classifications (1970). Primary 43 A07.

Key words and phrases. locally compact amenable group, compact group, invariant mean, topologically invariant mean, bounded Borel function.

${ }^{1}$ Supported in part by NSF grant MSC 76-06377A01. 


$$
\sup \{\mu(f): \mu \in \mathrm{LIM}\}=\inf _{A} \operatorname{ess} \sup _{x}\left\{\frac{1}{n} \sum_{i=1}^{n} f\left(a_{i} x\right)\right\},
$$

where the infimum is taken over all finite subsets $A=\left\{a_{1}, \ldots, a_{n}\right\}$ of $G$;

(3) for each $\theta \in \mathscr{D}$, the subspace $\left\{\rho_{\theta} f: f \in L^{\infty}(G)\right\}$ of $l^{\infty}(G)$ has a left invariant mean;

(4) for each permanently positive $(P P)$ set $E \subset G,\left\|\chi_{E}-h\right\|_{\infty} \geqslant 1$ if $h \in H$ $=$ the linear span of $\left\{l_{x} f-f: f \in L^{\infty}(G), x \in G\right\}$.

PP sets were first defined by Rudin [7]. Recall that a set $E \subset G$ is PP if it is Borel measurable and $\lambda\left(x_{1} E \cap \cdots \cap x_{k} E\right)>0$ for any given $x_{1}, \ldots, x_{k} \in$ $G$.

Let

$$
\begin{aligned}
\operatorname{TLIM}=\left\{\mu \in L^{\infty}(G)^{*}:\right. & \|\mu\|=1, \mu \geqslant 0 \\
& \text { and } \left.\mu(\xi * f)=\mu(f) \text { if } f \in L^{\infty}(G), \xi \in L^{1}(G),\|\xi\|_{1}=1, \xi \geqslant 0\right\},
\end{aligned}
$$

the set of topologically left invariant means on $L^{\infty}(G)$. It is known that TLIM $\subset$ LIM and if $G$ is amenable then TLIM $\neq \varnothing$, cf. [2]. Granirer [1] and Rudin [7] proved, independently, that if $G$ is nondiscrete and $G_{d}$ is amenable then LIM $\supsetneqq$ TLIM. In particular, if $G$ is an infinite compact group and $G_{d}$ is amenable then LIM $\supsetneqq\{\lambda\}=$ TLIM. It is still unknown whether LIM $\supsetneqq$ $\{\lambda\}$ for every infinite compact group.

In [1], Granirer proved that $(1) \Rightarrow(2)$ and that if $G$ is a nondiscrete group with (2) then LIM $\supsetneqq$ TLIM. He conjectured that (2) does not hold true in general if $G_{d}$ is not amenable. Our $(2) \Rightarrow(1)$ confirms his conjecture. In [7], Rudin proved that $(1) \Rightarrow(3)$ and $(1) \Rightarrow(4)$ and that LIM $\supsetneqq$ TLIM if $G$ is nondiscrete and if either (3) or $\left(4^{\prime}\right)$ holds where $\left(4^{\prime}\right)$ is:

(4') If $h \in H$ then, for each open dense set $U \subset G,\left\|\chi_{U}-h\right\|_{\infty} \geqslant 1$.

He then suggested that (3) and ( $\left.4^{\prime}\right)$ might hold true for every compact group. Our theorem shows that if $G_{d}$ is not amenable then both (3) and (4) fail for $G$. Since every open dense set is $\mathrm{PP},(4) \Rightarrow\left(4^{\prime}\right)$. We are unable to decide whether $\left(4^{\prime}\right) \Rightarrow(4)$ or whether $\left(4^{\prime}\right)$ holds for every compact group.

In [7], Rudin raised the question whether functions of the form $\rho_{\theta} f$ are Borel measurable. Wells [8] employed a nice Baire categorical argument to show that for every infinite compact metric group $G$ there exist $\theta \in \mathscr{D}$ and $f \in L^{\infty}(G)$ such that $\rho_{\theta} f \notin L^{\infty}(G)$. Rosenblatt [5] elaborated Wells' argument to obtain a stronger result: If $G$ is a nondiscrete metric group then for each $S \subset l^{\infty}(G)$, card $S \leqslant c$, there exists $\theta \in \mathscr{D}$ such that $\rho_{\theta}\left(L^{\infty}(G)\right) \supset S$. As a consequence, he gave the following:

TheOREM A (RosenblatT). Let $G$ be a nondiscrete $\sigma$-compact locally compact group. Then $G_{d}$ is amenable if and only if every nonempty closed invariant subset of $\mathscr{D}$ admits a G-invariant probability measure. 
Our proof depends on this theorem of Rosenblatt.

Proof of THE Theorem. Since $(1) \Rightarrow(2),(1) \Rightarrow(3)$ and $(1) \Rightarrow(4)$ are known, it remains to show that (2) $\Rightarrow(4),(3) \Rightarrow(4)$ and (4) $\Rightarrow(1)$.

$(2) \Rightarrow(4)$. Let $E$ be a PP set in $G$. Then, for each finite set $A=$ $\left\{a_{1}, \ldots, a_{n}\right\} \subset G$,

$$
\text { ess } \sup _{x}\left\{\frac{1}{n} \sum_{i=1}^{n} \chi_{E}\left(a_{i} x\right)\right\}=1 .
$$

Therefore, by (2), there exists $\mu \in$ LIM such that $\mu\left(\chi_{E}\right)=1$. (Recall that LIM is $w^{*}$-compact.) So, for each $h \in H, \mu\left(\chi_{E}-h\right)=1$. Since $\|\mu\|=1, \| \chi_{E}-$ $h \|_{\infty} \geqslant 1$.

$(3) \Rightarrow(4)$. Let $E$ be a PP set in $G$. As in [7], note that $I=\left\{f \in L^{\infty}(G)\right.$ : the zero set of $f$ is contained in $G \backslash E\}$ is a proper ideal. Therefore there exists $\theta \in \mathscr{D}$ such that $\theta=0$ on $I$. It implies that $\rho_{\theta}\left(\chi_{E}\right) \equiv 1$. By assumption, $\rho_{\theta}\left(L^{\infty}(G)\right)$ has a left invariant mean $\nu$. Let $h=\sum_{i=1}^{\infty}\left(l_{a_{i}} f_{i}-f_{i}\right) \in H$. Then

$$
\rho_{\theta}\left(\chi_{E}-h\right)=1-\sum_{i=1}^{n}\left(l_{a_{i}}\left(\rho_{\theta} f_{i}\right)-\left(\rho_{\theta} f_{i}\right)\right) .
$$

So $\nu\left(\rho_{\theta}\left(\chi_{E}-h\right)\right)=1$. Since $\left\|\rho_{\theta}\left(\chi_{E}-h\right)\right\|_{\infty} \leqslant\left\|\chi_{E}-h\right\|_{\infty}$ and $\|\nu\|=1, \| \chi_{E}$ $-h \|_{\infty} \geqslant 1$.

(4) $\Rightarrow(1)$. For each Borel set $B$ in a locally compact group $G$ there exists a closed-open set $\hat{B} \subset \mathscr{D}$ such that $\hat{\chi}_{B}=\chi_{\hat{B}}$; in fact, $\hat{B}=\left\{\theta \in \mathscr{D}: \theta\left(\chi_{B}\right)=1\right\}$. Sets of the form $\hat{B}$ form a topological basis for $\mathscr{Q}$. It is easy to check that $\left(B_{1} \cap B_{2}\right)^{\wedge}=\hat{B}_{1} \cap \hat{B}_{2},\left(B_{1} \cup B_{2}\right)^{\wedge}=\hat{B}_{1} \cup \hat{B}_{2}$ and $\left(x B_{1}\right)^{\wedge}=L_{x}\left(\hat{B}_{1}\right)$ if $B_{1}$ and $B_{2}$ are Borel sets in $G$ and $x \in G$.

Now assume that (4) holds for $G$. If $E$ is a PP set then by the Hahn-Banach theorem there exists $\mu \in \operatorname{LIM}$ such that $\mu\left(\chi_{E}\right)=1$ or, equivalently, $\hat{\mu}(\hat{E})=1$, i.e., supp $\hat{\mu} \subset \hat{E}$.

Let $K$ be a nonempty closed $G$-invariant subset of $\mathscr{D}$. Let

$$
\mathcal{E}=\{E: E \text { a Borel subset of } G \text { and } \hat{E} \supset K\} .
$$

Then $\cap\{\hat{E}: E \in \mathcal{E}\}=K$ and $\mathcal{E}$ is a downward directed set. If $E \in \mathcal{E}$ and $x_{1}, \ldots, x_{n} \in G$ then

$$
\begin{aligned}
\left(x_{1} E \cap \cdots \cap x_{n} E\right)^{\wedge} & =L_{x_{1}} \hat{E} \cap \cdots \cap L_{x_{n}} \hat{E} \\
& \supset L_{x_{1}} K \cap \cdots \cap L_{x_{n}} K=K .
\end{aligned}
$$

So $\lambda\left(x_{1} E \cap \cdots \cap x_{n} E\right)>0$ and hence $E$ is a PP set. By the last paragraph, for each $E \in \mathcal{E}$ we may choose $\mu_{E} \in$ LIM such that supp $\hat{\mu}_{E} \subset \hat{E}$. Let $\mu$ be a $w^{*}$-limit point of the net $\left\{\mu_{E}\right\}_{E \in \mathcal{E}}$. Since LIM is $w^{*}$-compact, $\mu \in$ LIM. If $\theta \in \mathscr{D} \backslash K$, then there exists $E \in \mathcal{E}$ such that $\theta \notin \hat{E}$. Therefore $\mu(\mathscr{D} \backslash \hat{E})=$ 0 and $\mathscr{D} \backslash \hat{E}$ is a neighborhood of $\theta$. So $\theta \notin$ supp $\mu$ and hence supp $\mu \subset K$. By Theorem A of Rosenblatt quoted above we conclude that $G_{d}$ is amenable.

REMARKs. (1) By applying Rosenblatt's result it is possible to prove that (3) $\Rightarrow(1)$ and $(2) \Rightarrow(1)$ directly. Conceivably there is a direct proof of the fact that $(4) \Rightarrow(2)$ without applying his result. 
(2) For a discrete group $G$, it is easy to see that a set $E \subset G$ is PP if and only if it is left thick in the sense of Mitchell [3]. Therefore, the following statement can be considered as a generalization of his Theorem 7: Let $G$ be a locally compact group, $G_{d}$ amenable. Then a Borel set $E \subset G$ is $P P$ if and only if there exists $\mu \in \mathrm{LIM}$ such that $\mu\left(\chi_{E}\right)=1$.

(3) Assume that $G$ is a $\sigma$-compact nondiscrete group. Then $P=\cap\{\hat{U}: \hat{U}$ open dense in $G$ \} is a nonempty $G$-invariant closed subset of $\mathscr{D}$. Therefore if $G_{d}$ is amenable then there exists $\mu \in \mathrm{LIM}$ such that $\hat{\mu}(P)=1$. In particular, $\mu\left(\chi_{U}\right)=1$ for each open dense set $U \subset G$. (Note that it is known that for each $\epsilon>0$ there exists an open dense set $U$ with $\lambda(U)<\epsilon$; see [1, Proposition 2].) If we assume further that $G$ is compact then $U \cap E$ is PP if $U$ is open dense and $E$ is PP, cf. [7]. Let $K$ be a minimal $G$-invariant subset of $\mathscr{D}$, i.e., $K$ is nonempty, closed and $G$-invariant and is minimal with respect to these properties. Then

$(\cap\{\hat{E}: E$ a Borel set, $\hat{E} \supset K\}) \cap(\cap\{\hat{U}: U$ open dense in $G\})$ is nonempty, i.e., $K \cap P \neq \varnothing$. By minimality of $K, K \subset P$. (This fact is also contained in the proof of Proposition 4.8 in [6].) Note that Rosenblatt [5] has shown that $\mathcal{D}$ has at least $2^{c}$ minimal $G$-invariant sets.

(4) For an infinite compact group $G$, the condition that $G_{d}$ is amenable is not a necessary condition for LIM $\supsetneqq\{\lambda\}$. First let us give the following.

LeMMA. Let $G$ be a compact group and $N$ a closed normal subgroup of $G$. Then there is a continuous affine embedding of $\operatorname{LIM}(G / N)$ into $\operatorname{LIM}(G)$.

Proof. If $f \in L^{\infty}(G)$ let $\tilde{f} \in L^{\infty}(G / N)$ be defined by

$$
\tilde{f}(\dot{x})=\int_{N} f(x t) d \lambda_{N}(t), \quad x \in G .
$$

Here $\dot{x}$ is the coset $x N$ and $\lambda_{N}$ is the normalized Haar measure of $N$. It is easy to check that $\tilde{f}$ is indeed a well-defined function in $L^{\infty}(G / N)$ and that $\|\tilde{f}\|_{\infty} \leqslant\|f\|_{\infty}$.

If $\nu \in \operatorname{LIM}(G / N)$, define $\tilde{\nu} \in L^{\infty}(G)^{*}$ by $\tilde{\nu}(f)=\nu(\tilde{f}), f \in L^{\infty}(G)$. It is easily checked that $\|\tilde{\nu}\|=1, \tilde{\nu} \geqslant 0$ and, if $x \in G$ and $f \in L^{\infty}(G)$,

$$
\tilde{\nu}\left(l_{x} f\right)=\nu\left(\left(l_{x} f\right)^{\sim}\right)=\nu\left(l_{\dot{x}}(\tilde{f})\right)=\nu(\tilde{f})=\tilde{\nu}(f) .
$$

Therefore, $\tilde{v} \in \operatorname{LIM}(G)$. Since $f \rightarrow \tilde{f}$ sends $L^{\infty}(G)$ onto $L^{\infty}(G / N)$, the mapping $\nu \rightarrow \tilde{\nu}$ is one-to-one.

By the above lemma, we know that $\operatorname{LIM}(G) \supsetneqq\{\lambda\}$ if $G=\operatorname{SU}(2) \times \mathbf{T}$ (direct product). The following example is perhaps more interesting.

EXAMPLE. A theorem of Iwasawa states that a free group is residually a finite $p$-group (for all primes $p$ ) (cf. [4, p. 117]). Therefore, if $F$ is the free group on two generators then there exists a sequence of normal subgroups $\left\{F_{n}\right\}$ of $F$ such that $F \supsetneqq F_{1} \supsetneqq F_{2} \supsetneqq \ldots, \cap_{n=1}^{\infty} F_{n}=\{1\}$, and for each $n$, $F / F_{n}=G_{n}$ is a finite $p$-group. Then $F$ can be considered as a subgroup of $G=\Pi_{n=1}^{\infty} G_{n}$ (complete product). Hence, with product topology $G$ is compact 
and $G_{d}$ is nonamenable. We claim that $\operatorname{LIM}(G) \supsetneqq\{\lambda\}$. Indeed for each $n$, choose a (proper) maximal subgroup $N_{n}$ of $G_{n}$. Then $\left[G_{n}: N_{n}\right]=p$ and $N_{n}$ is normal in $G$. Let $N=\prod_{n=1}^{\infty} N_{n}$. Then $N$ is a compact normal subgroup of $G$ and $G / N \cong \prod_{n=1}^{\infty}\left(G_{n} / N_{n}\right)$ is an infinite abelian compact group. By the above $\operatorname{lemma} \operatorname{LIM}(G) \supsetneqq\{\lambda\}$.

\section{REFERENCES}

1. E. Granirer, Criteria for compactness and for discreteness of locally compact amenable groups, Proc. Amer. Math. Soc. 40 (1973), 615-624.

2. F. P. Greenleaf, Invariant means on topological groups and their applications, Van NostrandReinhold, New York, 1969.

3. T. Mitchell, Constant functions and left invariant means on semigroups, Trans. Amer. Math. Soc. 119 (1965), 244-261.

4. D. J. S. Robinson, Finiteness conditions and generalized soluble groups, Part 2, SpringerVerlag, Berlin, 1972.

5. J. M. Rosenblatt, Invariant means for the bounded measurable functions on a non-discrete locally compact group, Math. Ann. 220 (1976), 219-228.

6. Invariant means and invariant ideals in $L_{\infty}(G)$ for a locally compact group $G, \mathrm{~J}$. Functional Analysis 21 (1976), 31-51.

7. W. Rudin, Invariant means on $L^{\infty}$, Studia Math. 44 (1972), 219-227.

8. B. Wells, Homomorphisms and translates of bounded functions, Duke Math. J. 41 (1974), 35-39.

Department of Mathematics, State University of New York at Buffalo, Buffalo, New YORK 14222 